\title{
Protée
}

\section{Comment faire des nations avec des signes. L'hypoicône et la genèse des communautés imaginées}

\section{Fernando Andacht}

Volume 26, numéro 3, 1998

Logique de l'icône

URI : https://id.erudit.org/iderudit/030526ar

DOI : https://doi.org/10.7202/030526ar

Aller au sommaire du numéro

Éditeur(s)

Département des arts et lettres - Université du Québec à Chicoutimi

ISSN

0300-3523 (imprimé)

1708-2307 (numérique)

Découvrir la revue

Citer cet article

Andacht, F. (1998). Comment faire des nations avec des signes. L'hypoicône et la genèse des communautés imaginées. Protée, 26(3), 55-63.

https://doi.org/10.7202/030526ar
Résumé de l'article

L'article revient sur la recherche entreprise par B. Anderson sur la communauté imaginée à la lumière de la conception peircienne de la qualité incarnée, ou hypoicône. L'analyse de la naissance des nations modernes révèle des implications insoupçonnées dues à la tension entre la qualité pure et son incarnation matérielle. L'article a pour but d'illustrer le potentiel analytique de la recherche sémiotique en ce qui concerne le fonctionnement de l'imaginaire. 


\section{COMMENT FAIRE DES NATIONS AVEC DES SIGNES L'HYPOICÔNE ET LA GENĖSE DES COMMUNAUTÉS IMAGINÉES}
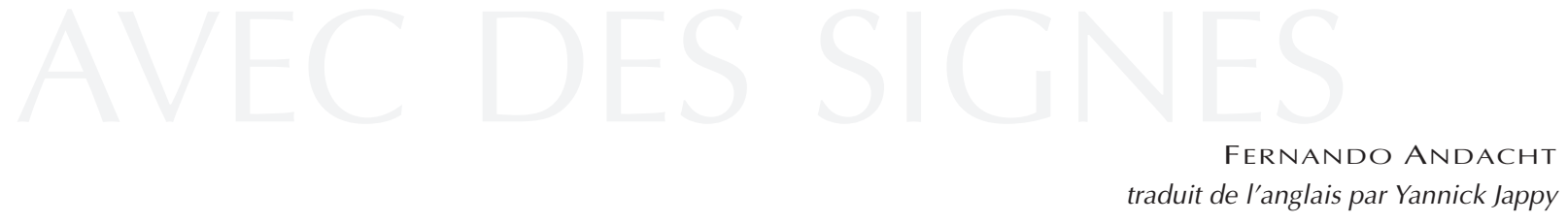

LE CHEMIN ICONIQUE DE LA CONNAISSANCE, OU COMMENT RÊVER DES RÉALITÉS NOUVELLES

Selon la «règle de l'instanciation " 1 , tout signe, qu'il soit icône, indice ou symbole, doit être actualisé pour fonctionner comme tel. Il n'y a donc rien d'étonnant en apparence à ce que le signe de la qualité ou qualisigne - porteur d'une ressemblance potentielle avec tout objet qu'il peut iconiser - soit considéré dans ses manifestations effectives comme une hypoicône (C.P. 2.276). Cependant, l'étude de la fonction épistémique d'un signe iconique (cf. Ransdell, 1979) peut enrichir de façon inattendue un aspect moins connu de la sémiotique de Peirce, à savoir sa conception de l'imagination humaine. Alors qu'associer sens et action s'impose comme une évidence pour le pragmatisme, le lien est moins évident avec «les dimensions créative, temporelle et expérimentale de l'expérience» (Alexander, 1990: 325). Donner son dû à ce courant de pensée passe par une «reconnaissance du rôle d'un mode de compréhension dans lequel le réel est lu et reconstruit à la lumière du possible» (ibid.); c'est cette reconnaissance que nous souhaitons mettre en évidence dans cet article.

Pour cela nous nous appuierons sur l'analyse de l'iconicité de Joseph Ransdell ${ }^{2}$ pour revenir ensuite sur l'étude classique d'Anderson (1983) de la communauté imaginée, dont il fait le pivot du type de nationalisme qui s'est développé dans des colonies d'Amérique ou d'Asie au 19e siècle. Nous espérons ainsi montrer les possibilités théoriques de nouvelles implications sociologiques de la sémiotique générale de Peirce, but que nous avons déjà poursuivi dans une étude approfondie de la notion théorique très controversée de «fondement» (cf. Andacht, 1996) dans son rapport avec l'imaginaire social.

\section{L'APPÂT DE L'HYPOICÔNE: RÊVER DE TARTES AUX POMMES ET DE TABLEAUX}

Le propre des hypoicônes est de nous montrer la réalité en «donnant corps à leurs objets tels qu'ils sont en eux-mêmes, vus sous un certain angle» (Ransdell, [1986] 1997: § 50), c'est aussi leur attrait irrésistible. Aussi bizarre que cela puisse paraître, pour parler de fonction épistémique, la notion de "rêve» est bien au centre de la conception peircienne de la révélation iconique. Laissons de côté, 
parmi les nombreux emplois du mot «rêve» dans les Collected Papers, les emplois littéraux ainsi que celui métaphorique de "fantastique» ou "faux", et il nous reste encore un fond non négligeable d'utilisations analogiques du terme pour décrire le fonctionnement des qualisignes tels qu'ils sont réalisés dans les hypoicônes. Avant d'en citer des exemples précis, nous voulons introduire une sorte de cas «intermédiaire», parce qu'il montre ce qui a pu attirer l'attention de Peirce sur l'usage métaphorique précité. Peirce décrit la réalité comme «la manière persistante dont les choses s'imposent à notre reconnaissance» (C.P. 1.171), phénomène dont l'absence est donc un signe fiable que quelque chose n'est qu'un «simple rêve» (ibid., les italiques sont de Peirce). L'hypoicône est justement le résultat de l'association de cette "insistance" matérielle avec le caractère imaginaire, irréel, onirique des qualités; c'est une «image insistante», selon le terme de Peirce (C.P. 8.104), pour décrire le but ultime de l'enquête scientifique.

Nous allons maintenant chercher des exemples de l'emploi plus technique du mot «rêve» dans un passage où Peirce décrit longuement et de façon allégorique l'interaction des trois catégories phanéroscopiques. Rien de plus américain que l'apple-pie (tarte aux pommes). Ainsi semble le penser Peirce lorsque, pour "examiner l'idée de généralité " ${ }^{3}$, il choisit d'utiliser une voix passive inhabituelle pour commencer sa recette détaillée de ce dessert typiquement américain: «une tarte est désirée». Choix syntaxique marqué pour mieux montrer le goût général pour ce dessert, comme si Peirce disait: il est tout à fait normal que tout Américain en désire une. Suit alors une remarque contraire à l'intuition: «nous désirons rarement, peut-être jamais, une seule chose individuelle». Peirce explique que le désir est lié au plaisir, «lui-même une qualité». Nous avons ici de purs premiers, des qualisignes, qui en tant que tels sont tout à fait différents de cas particuliers et réels de plaisir, où quelqu'un, quelque part, à un moment donné, mange vraiment une tarte. Au centre de notre plaisir de consommateur potentiel il y a, dit Peirce, notre aptitude à «imaginer», et ceci vaut aussi pour la cuisinière à qui on demande de faire cette tarte. Consommateur et cuisinière doivent partager ce «rêve». Bien qu'elle ne puisse que "choisir telle ou telle pomme" pour sa recette, «elle poursuit une idée ou un rêve sans aucune idée préconçue quant à son heccéité. Toute simple et concrète qu'elle semble, la confection de la tarte, puisqu'elle relève de la nature du rêve, est totalement irresponsable; elle est ce qu'elle veut» ${ }^{4}$.

Ainsi analysés, les rêves, bien qu'étant par nature évanescents, ont bien une fonction importante dans notre vie: ils jouent le rôle de vision médiatrice entre l'événement singulier, unique, et la règle générale, valable, elle, pour longtemps comme, dans ce cas précis, norme culinaire. Ainsi le drame de notre vie se joue-t-il sur une scène réelle et tient-il compte par exemple de la règle des trois unités comme des indications du metteur en scène, mais il reste soumis au pouvoir invisible de notre imagination créatrice. Une tarte aux pommes, de grandes nations en train de naître, ou des symboles verbaux ${ }^{5}$, tous reposent au départ sur un rêve constructif, excluant toute heccéité.

Un autre exemple d'image rêvée servant à illustrer les icônes est la description du genre d'objet qui vient à l'esprit dès que nous pensons à la ressemblance qui lie le signe et son objet. La contemplation intense d'un tableau peut nous mener à un état proche du rêve $^{6}$, où la frontière entre réalité et apparence est très floue: «au moment où nous perdons conscience du fait que ce n'est pas la chose elle-même, la différence entre réalité et copie disparaît» (C.P. 3.362). Ce que Peirce présente comme le «rêve pur de la contemplation d'une icône» (ibid.) n'est-il pas l'une des expériences les plus familières de notre modernité? Qui n'a jamais été ainsi fasciné en regardant un film avec d'autres étrangers anonymes dans une pièce sombre comme le ventre maternel? Ransdell remarque que ce qui est la marque distinctive des signes iconiques tels que celui que donne Peirce dans l'exemple ci-dessus (cela vaut aussi pour cartes et photos, etc.), c'est que les icônes qu'ils incorporent renvoient à deux référents, puisqu'elles ont un sens en elles-mêmes, mais renvoient aussi à un «autre» du fait 
qu'elles révèlent un objet qui, souvent ou généralement, n'est pas immédiatement accessible - le pays représenté dont la carte est l'hypoicône (Ransdell, 1997 : §55). C'est là l'attrait de l'hypoicône: rendre physiquement réel ce qui ne peut être touché ou vu dans le monde extérieur (l'objet de l'hypoicône). L'attirance exercée sur les hommes par l'hypoicône, et son succès, tient à ce va-et-vient entre les éléments séparés que sont la promesse du rêve et sa matérialité discrète mais réelle. Ce trait est une variation iconique sur la suspension du doute (suspension of disbelief) que présuppose le plaisir des textes littéraires, ce que nous appellerons volontiers la «fonction transparente» des hypoicônes. Un doute subsiste: que faire du fait qu'aucun signe "ne peut agir en tant que tel tant qu'il n'est pas incarné» (C.P. 2.244), alors que cette «incarnation n'a rien à voir avec son caractère en tant que signe»(ibid.)? Que faire de l'opposition entre la nature qualitative de l'icône et sa matérialité? Pour paraphraser une question célèbre, nous devons peutêtre nous demander ici "qu'y a-t-il dans un corps?». Cette question de l'incarnation est-elle banale, le paramètre par défaut du signe, ou bien quelque chose de riche en implications importantes pour la nature de la sémiose iconique? Pour apporter quelques éléments de réponse à cette question, nous proposons un récapitulatif de l'emploi technico-métaphorique du terme «rêve" chez Peirce:

Rêve-Qualité-Imaginaire-Désir Inassouvi:

la Priméité comme principe de la mise en forme.

Expérience-Singulier-Hecceité-Délimitation concrète: la Secondéité comme force de la matérialisation.

Ransdell (1979) suggère que ce mode iconique de la sémiose nous fournit le moyen d'échapper au piège idéaliste kantien, à cette pierre d'achoppement du Ding an sich. "L'opinion finale», très controversée, que Peirce appelle aussi «l'idée finale prédestinée» (C.P. 8.103), est intimement liée à sa dimension physique, «l'aspect de sinsigne de l'icône incarnée» (Ransdell, 1979: 63) décrit par Peirce comme «une image insistante» (C.P. 8.104) pour nous aider à comprendre l'approche progressive scientifique de la vérité. On peut poser, dans notre perception du monde, une identité quasiment parfaite entre un signe iconique et son objet; en voici deux exemples.

\section{PROUESSES QUOTIDIENNES DE L'IMAGINATION ET POUVOIR DE L'HYPOICÔNE}

Dans une anecdote familiale racontée deux fois et qui met en scène Herbert, jeune frère de Peirce, nous trouvons un exemple du fonctionnement de la dualité icône/hypoicône dans une manifestation de l'imagination. Un jour, la mère (une voisine, dans la version de C.P. 5.487, n1)

renversa de l'alcool enflammé sur sa jupe. Instantanément, avant qu'aucun de nous ait pu penser à faire le moindre geste, mon frère, Herbert, tout petit à l'époque, avait saisi un tapis et éteint le feu. Nous fûmes étonnés de cette promptitude qui devait le caractériser adulte. Je lui demandai comment l'idée lui était venue aussi vite. Il répondit: J'avais envisagé auparavant ce que je ferais dans le cas où un tel accident se produirait.

(C.P. 5.538)

Peirce utilise cet incident pour analyser les mécanismes de notre imagination dans la vie et dans la science. Le lien sémiotique entre notre façon d'imaginer et d'agir accroît la généralité dans le monde. La réaction et la réponse d'Herbert sont deux exemples de ce que Peirce appelle "une résolution», c'est-à-dire "l'acte d'approuver, de "faire sienne", une ligne de conduite imaginaire, de sorte qu'elle puisse influer sur notre conduite effective ultérieurement" (ibid.). Ces «riens éthérés, désincarnés» (C.P. 6.455) que sont nos rêves portent vraiment des fruits dans le monde extérieur; et Peirce présente les icônes comme "le fait d'envisager comment j'agirais dans tel cas, comme modèle de la conduite possible d'un autre» (C.P. 2.92). Nous voyons dans cet incident le rapport entre les deux mondes dans lesquels «nous vivons»: "un monde de faits» et "un monde de rêves» (C.P. 1.321). Meublant en partie ce royaume des faits, il y a les hypoicônes qui, comme vision d'un futur possible, permettent à l'enfant de répéter son geste au cas où un tel accident se produirait un jour; elles lui ont permis de prévoir - mais pas de prédire - l'avenir (cf. Ransdell, 
1979: 61). Décisive aussi dans l'acte accompli par le frère de Peirce est la pure qualité possible du sentiment d'héroïsme, icône même de l'image impressionnante où l'on sauve son prochain de façon aussi courageuse et rapide.

Mais, comment expliquer que, devant ce même objet ordinaire, visible de tous, seul l'enfant imagine, anticipe ce fait original qu'est le danger potentiel de cette lampe et les moyens les plus adéquats à prendre en cas d'urgence? Cette attitude mérite le nom d'imagination préparatrice, et pour Peirce, elle nous différencie des autres créatures ${ }^{7}$. Ce qui permet à cette vision d'un futur possible de se réaliser, c'est cette qualité changeante qui prend corps par la suite dans la préparation physique et mentale accomplie par le jeune Herbert avant l'acte qui suscite l'admiration du sémioticien. Lorsqu'enfin ce signe s'accomplit, il produit une autre hypoicône, l'acte de bravoure de l'enfant, conservé comme souvenir émerveillé par son frère. De ces hypoicônes découlent de nouvelles règles de sens et d'action, telle la promptitude à secourir quelqu'un en difficulté, phénomène qui a le caractère d'une loi et qualité que gardera plus tard l'enfant. Notre intérêt va au rapport entre les deux premiers éléments, la vision qualitative intangible de ce qui est appréhendé et manipulé (intérieurement puis extérieurement) grâce aux hypoicônes de la vision et de la mémoire, pour être finalement "faite sienne" (C.P. 5.538).

Voyons un autre exemple d'un lien entre la possibilité apparemment totalement aléatoire des icônes par opposition à leur manifestation concrète en hypoicônes, pris dans le genre d'histoires drôles populaire chez nous, en Uruguay, et peut-être aussi ailleurs. La structure en est simple:

En quoi $X$ ressemble-til à $Y$ ?

Dans une telle question, «X» est en général quelqu'un de connu, disons un homme politique, le plus souvent impliqué dans un problème fort discuté, de préférence controversé, ou présenté ainsi par les médias. Quant à "Y», il représente un élément sans rapport avec « $X$ ». Ce pourrait être un artefact quelconque, une plante ou toute autre création de l'imagination fertile de l'humoriste anonyme. Dans une période politique difficile telle que la dictature militaire qui vit le jour dans les années soixante-dix en Uruguay, ce genre de blague iconique est particulièrement riche, révélant l'utilité de son mode d'expression pour ce qui ne peut être dit ouvertement. Le principe à la base de ce genre d'histoire n'est pas très éloigné de la maxime métaphorique d'Aristote, à savoir que la valeur de ce trope doit être mesurée par la distance sémantique entre l'élément métaphorisé et le support de la métaphore. En tant que signes de possibilité qualitative, les icônes nous permettent d'associer à notre gré deux éléments, quels qu'ils soient et quel que soit notre désir sémiotique, puisque dans le domaine de la ressemblance iconique tout est possible. Le destinataire de l'histoire sait d'avance qu'une hypoicône le guette sous la forme d'une image inattendue, donc amusante, reposant sur un point commun, très improbable certes, mais possible, entre "X» et «Y». L'effet iconique, qui est presque onirique, découle de notre quête éperdue d'éventuelles qualités analogiques, ou de l'impossibilité où nous sommes de trouver ce point commun, ainsi que l'invention préalable de la blague. La marge séparant icône et hypoicône nous permet d'appréhender le travail créatif de l'esprit humain, l'invention d'analogies nouvelles, drôles ou non, parfois brillantes, qui iront ensuite enrichir le répertoire d'hypoicônes déjà existantes.

La prouesse du jeune Herbert Peirce ainsi que le talent nécessaire à l'humoriste pour proposer une relation iconique inhabituelle, donc amusante, entre un personnage important et à peu près tout et n'importe quoi d'autre sont liés au fonctionnement de l'icône, à ce rêve pur par lequel notre monde nous apparaît sous un jour nouveau, impossible à entrevoir sans lui. L'hypoicône, que ce soit l'anecdote familiale de Peirce ou l'histoire drôle, est une cristallisation de cette créativité, preuve indiciaire du travail de l'icône elle-même, de ces qualités fluctuantes, insaisissables, indépendantes, qui jouent un rôle dans notre vie lorsqu'elles se projettent dans l'avenir par des manifestations concrètes. Voyons maintenant le rôle 
joué par les icônes dans la genèse d'institutions durables, puissantes, telles que les nations ou les révolutions sociales, c'est-à-dire dans le processus de l'imaginaire collectif.

\section{LE POUVOIR DE L'HYPOICÔNE}

COMME INSTRUMENT DE LIBÉRATION NATIONALE

L'intérêt particulier de l'analyse qu'Anderson (1993) fait de l'émergence de nations modernes au 19 e siècle dans d'anciennes colonies européennes d'Amérique ou d'Asie tient au rôle-clef qu'il attache à la qualité «imaginaire/imaginée» de ces constructions sociales. Si nous laissons de côté le caractère psychologiste donné par l'allusion à l'esprit des fondateurs de nations, la description suivante nous présente un monde non dénué de rapport avec la sémiose iconique:

[C']est une communautéimaginée, et imaginée comme à la fois intrinsèquement limitée et souveraine. Elle est imaginée dans la mesure où les membres de la plus petite nation ne se connaîtront jamais, ne se rencontreront jamais, n'entendront jamais parler des autres, et pourtant chacun d'eux porte présente à l'esprit l'image de cette communion.

(Anderson, 1993: 6sq.; je souligne)

Les hypoicônes (et les icônes) contribuent à la création de nations à deux titres. On nous dit d'abord que le sentiment «de profonde camaraderie transversale» (ibid.: 7), qui définit la nation pour ses membres et qui est donc décisive pour la création d'une telle communauté, n'est qu'une image. De plus, si nous en croyons Anderson (ibid.: 118-132), certaines hypoicônes utilisées par les colonisateurs comme outils pédagogiques pour "former» les colonisés jouent un rôle dans la naissance de ce sentiment. Certaines qualités matérialisées, comme les cartes, les photos, les monuments et les hymnes nationaux qui correspondent aux icônes à «double référent» de Ransdell, contribuent à la création de toutes pièces de ces objets que sont les nouvelles nations. Ainsi le mouvement indépendantiste créole en Amérique ou en Asie peut-il être vu comme l'effet sémiotique de ces hypoicônes. Ce résultat inattendu peut aussi être le fruit de l'utilisation d'une hypoicône atypique, telle que les journaux, une nouveauté à l'époque ${ }^{8}$. Ce médium imprimé leur donnait un «lieu» typographique où, pour la première fois, les colonisés pouvaient se voir avec leurs pairs, en de mêmes lieux et temps; l'effet était fort exaltant pour eux. Bien que, d'un point de vue technique, en tant qu'hypoicônes les cartes en couleurs soient des diagrammes (cf. C.P. 2.277) et que les journaux soient remplis de répliques de symboles, ces hypoicônes fonctionnent en tant qu'images chez Anderson, puisqu'elles incarnent la qualité de «fraternité transversale», qualité indispensable dans la naissance d'une nation.

Ces signes créent un sentiment de communion nationale dans ce qui n'était jusque-là qu'un regroupement hétérogène, accidentel, de gens sans traditions et parfois même sans langue communes, l'indonésien Bahasa, par exemple (Anderson, 1993: 132), dépourvu bien entendu de la notion moderne du partage dans un territoire commun de ce plafond sémiotique que sont un drapeau et un hymne national. Ils transforment ainsi la réalité brutale de la colonisation en un rêve d'autonomie politique. Tel est bien le pouvoir étonnant de ces hypoicônes, ces «imaginations limitées», comme les appelle Anderson dans son étude; elles incorporent des éléments instables comme les rêves et leur confèrent la stabilité, l'immuabilité de la loi. Ce tour de passe-passe donne naissance à la puissance parfois terrifiante du nationalisme, qui s'exerce sur des millions de gens qui sont prêts «moins à tuer qu'à [...] mourir pour de telles imaginations limitées» (ibid.).

L'étrange effet des icônes sur les objets qu'elles nous donnent à voir, c'est de les rendre presque «purement imaginaires» (C.P. 4.448). Pareillement, Anderson refuse toute idée de faux - et a fortiori de vrai - dans les images dont il parle. Il n'admet pas qu'on analyse «l'invention» d'une nation comme une "pure fabrication", puisque son étude doit se comprendre comme la défense de la notion d'«imaginer ou de créer» 9 .

Question cruciale posée par Anderson: «pourquoi est-ce justement les communautés créoles qui ont 
développé si tôt ce sentiment de nation constituée bien avant la plupart de l'Europe?» (Anderson, 1993:

50). Dans sa recherche d'une réponse, nous trouvons la preuve de la pertinence de l'hypoicône et de l'icône dans la création de cette notion capitale dans l'histoire de la modernité. Nous ne pouvons pas manipuler rêves et qualités à notre gré mais, Peirce le démontre, ces qualités influencent nos actes en donnant une image agréable ou désagréable de certains aspects de la vie, amenant ainsi des changements temporaires ou durables dans nos vies personnelles. Les peuples colonisés décrits par Anderson ne peuvent pas souhaiter tel ou tel type de nation (cf. C.P. 1.341 et la discussion ci-dessus), en regardant simplement les cartes en couleurs de leur école coloniale. Ils peuvent cependant se prendre à rêver à certaines qualités nouvelles - un début de fraternité, fraternité qui rend la lutte pour l'indépendance si attirante. Une partie de cette lutte tient au sentiment d'appartenance à un royaume inconnu, à la communauté imaginée appelée Indonésie ou Pérou. Ceci implique parfois l'adoption d'une langue nouvelle telle que l'indonésien Bahasa, dont l'existence est liée à son utilité pour le colonisateur ${ }^{10}$. Nous voulons maintenant revenir sur le problème de la matérialisation iconique en considérant l'aspect indiciaire, lui aussi partie intégrante de la construction imaginaire d'une nation; nous allons placer ce rêve colonial dans une salle de classe.

Anderson (1993: 132sqq.) insiste sur l'importance pour de futurs patriotes et nationalistes d'avoir simplement étudié ensemble dans la même école coloniale. Cette éducation devait être un privilège, le début de "pèlerinages" (ibid.: 121) communs autorisés aux membres d'une intelligentsia indigène, lancés sur le chemin d'une ascension sociale restreinte et limitée par les colonisateurs. Cette coexistence physique a permis à des hommes sans aucun lien ethnique ou culturel de se sentir et de se considérer "patriotes" et «compatriotes». On voit ici la dimension indiciaire, le sinsigne ou l'aspect matérialisé du processus de création du sens. Seul l'indice, écrit Peirce (C.P. 4.448), est capable de donner cette «assurance d'un fait positif». C'est ce que stipule la règle de l'inclusion: une nation est imaginée mais sa genèse est ancrée dans une circonstance unique comme la classe décrite ci-dessus. Même une activité ordinaire comme chanter ensemble - à l'unisson, en quelque sorte - ${ }^{11}$ a une pertinence sémiotique pour le mouvement de libération ${ }^{12}$.

"C'est la magie du nationalisme que de transformer le hasard en destinée» (Anderson, 1993: 12). Un regard sémiotique porté sur ce jugement quelque peu laconique d'Anderson sur la création des nations nous permet d'en fournir une explication plus riche. "L'alchimie» nationaliste associe l'aléatoire de la priméité, catégorie de l'indéterminé, de ce qui est tel qu'il est indépendamment de toute autre chose, avec la prévisibilité inhérente aux symboles, tournés vers l'avenir et se comportant comme des lois, et ce grâce au support essentiel qu'est la matérialisation de ces signes, ancrés dans un lieu et un temps.

\section{L'HYPOICÔNE COMME OUTIL DE LA RÉVOLUTION: LE MYTHE DANS LE TRAVAIL DE GEORGES SOREL}

Nous voulons conclure ces réflexions sur l'hypoicône en revenant brièvement sur un autre type de "communauté imaginée», qui s'est développée dans le partage de mythes sociaux, et qui fut décrite au début du 20e siècle par Georges Sorel (1908) dans une étude originale des mouvements sociopolitiques. Sorel essaie de démontrer que la révolution ouvrière préconisée par le marxisme ne peut s'accomplir que si l'on abandonne les mots et les discussions pour ne garder que les mythes entretenus par les ouvriers, en particulier celui de «la grève générale» (ibid.: 32 sq.). Les réflexions de Sorel sur la lutte des classes se rapprochent beaucoup de la description que fait Peirce du fonctionnement de l'hypoicône en relation avec l'imagination humaine.

Sorel insiste, s'emporte même, pour refuser d'analyser avec des mots les conditions d'un tel mythe de la grève générale, puisque sa valeur politique tient précisément au fait que c'est un élément purement qualitatif capable de pousser à des luttes héroïques les ouvriers qui le gardent à l'esprit, leur permettant de 
créer le genre de société nouvelle décrite dans les utopies socialistes irréalisables. Qu'importe, dit Sorel, si elle ne se produit pas vraiment, c'est-à-dire si les rues ne sont pas réellement aux mains de tous les ouvriers qui refusent par là les conditions inhumaines, les salaires de misère; envisager l'image purement visuelle, mentale d'un tel événement - la possibilité qualitative peircienne - doit suffire à pousser les masses vers une vraie rébellion. À ce moment-là seulement pourrontelles s'organiser et former une réelle force politique, capable de changer la société. L'élan de l'imagination donnera des ailes à l'action vraie, historique, politique. Sans lui l'action n'aura pas lieu. La description des mythes sociaux de Sorel est intéressante dans la mesure où elle ressemble fortement à la conception de l'hypoicône dans la sémiotique de Peirce:

[L]es hommes qui participent aux grands mouvements sociaux, se représentent leurs actions prochaines sous forme d'images de batailles assurant le triomphe de leur cause. Je proposais de nommer mythes ces constructions [...]: la grève générale des syndicalistes et la révolution catastrophique de Marx sont des mythes [...] je voulais montrer qu'il ne faut pas chercher à analyser de tels systèmes d'images, comme on décompose une chose en ses éléments, qu'il faut les prendre en bloc comme des forces historiques, et qu'il faut surtout se garder de comparer les faits accomplis avec les représentations qui avaient été acceptées avant l'action. (Ibid.: 32)

Ces images étant des qualisignes, elles ne présupposent aucun autre signe ou catégorie plus simple qu'elles et ne doivent donc pas être analysées pour y trouver un sens caché.

C'est ce que dit Peirce à propos de ces «signes originaliens" (C.P. 2.92) qui n'autorisent pas cette analyse, qui sont, pour ainsi dire, sans profondeur et n'ont forcément aucune autre détermination. Je suis ce que vous voyez ou entendez ou - mieux encore - ce que vous imaginez, nous dirait l'icône si elle pouvait parler. Pour Sorel, la dimension existentielle de ce mythe, ses aspects économiques et politiques sont secondaires, mais son pouvoir en tant qu'image est immense, permettant comme il le fait d'obtenir la libération des ouvriers de leurs oppresseurs. À partir d'une vision fondée sur l'ouï-dire, les émotions ou l'expérience d'une autre grève, partielle, les ouvriers peuvent arriver à se voir avec émotion comme protagonistes de ce grand mouvement, de cette Grève Générale future mais possible. Ainsi, ils seront prêts à affronter les durs obstacles d'une grève réelle, générale ou pas.

Peirce fait un récit quasiment poétique de nos souvenirs, des visions du passé qui reviennent nous hanter ou nous pousser à l'action héroïque, comme son frère ou les ouvriers de Sorel, fort pertinente ici:

La mémoire ne serait qu'un rêve si des prédictions qu'elle inspire ne se réalisaient pas. Si nous tenons compte $d u$ fait que ce sont d'infimes parcelles confuses de sentiment qui font la mosaïque de la mémoire, on est contraint de la comparer à la conjecture. C'est une merveilleuse force de construction de quasi-conjectures ou de rêves qui seront vérifiés par l'expérience future.

Nous voulons avancer ici l'idée que le mythe social de Sorel est une de ces «quasi-conjectures» ou un de ces « rêves» qui déterminent notre avenir en s'associant à des actions qui portent le nom général (symbole) de "grève générale». Le «mythique» ainsi compris a cette «douce compulsion» (ibid.), selon l'heureuse expression que Peirce utilise pour rendre compte du «pouvoir que le passé a sur nous» (ibid.). Il n'y a cependant rien de "doux" dans cette image révolutionnaire pour Sorel: elle est «tellement motrice qu'elle libère les hommes une fois qu'elle pénètre dans leurs esprits» (1908: 184). En partageant ce "rêve», terme pris dans le sens déjà vu, les ouvriers de l'étude de Sorel peuvent devenir membres d'une puissante communauté mythique, donc imaginée, composée de tous ceux qui ont présente à l'esprit cette vision exaltante de masses laborieuses en lutte, et qui donc peuvent agir en conséquence. Autre type d'unisson.

Résumons maintenant les divers points de contact entre cette théorisation précoce d'une notion qui allait devenir presque une subdivision des sciences sociales dans la deuxième moitié du 20 e siècle. Pour Sorel, le mythe c'est: 
a) une image ou un ensemble d'images;

b) une construction humaine liée à une époque ou un âge de l'humanité;

c) quelque chose qui ne devrait pas être analysé séparément, mais pris in toto faute de quoi son mode d'être s'en trouve déformé et donc détruit;

d) un plan d'action qui présente une vision du déjà accompli comme guide fiable pour l'action à venir.

Sorel pense que ces mythes sont des produits de la société, marqués par les conditions de vie de ceux qui les contemplent, sans rien de magique ou de mystique. Dans ce cas-là, le mythe semble être plutôt un produit de la manipulation des structures sociales, même s'il fonctionne de façon inconsciente, comme stratégie de défense «naturelle» d'un groupe donné. Le troisième point est l'un des plus intéressants: Sorel attaque toute analyse possible, toute réflexion prudente et rationnelle sur ces constructions mentales. Pourquoi un intellectuel met-il ses lecteurs en garde avec véhémence contre une telle attitude à l'égard des mythes? Simplement parce qu'ils sont monadiques, non décomposables, et que, sous le scalpel froid de la raison, ils se désintègrent et ne nous laissent qu'un arrière-goût de "rêve pur ", métaphore de Peirce (C.P. 3.386) pour la notion technique d'une icône proprement dite.

Pour Sorel, le mythe est un exemple de "l'image insistante» de Peirce (C.P. 8.104), une icône matérialisée (hypoicône) médiatrice du réel devenu instantanément accessible. Ceci est très différent de l'effet sémiotique produit par la tiercéité, par les mots capables d'analyser de façon théorique et pratique l'organisation d'une grande grève. Ce n'est que par le mythe que sera atteint le but ultime de la lutte des classes, écrit Sorel, cette "réforme de la volonté» (1908: 177), lorsque nous laisserons fonctionner librement les forces qui constituent les mythes sociaux. Nous voulons conclure cette section en soulignant un autre parallélisme remarquable entre les «images insistantes» de Peirce et la façon dont Sorel décrit la manière dont ces déterminations mythiques agissent sur l'imagination humaine:
Ces tendances viennent se présenter à l'esprit avec l'insistance d'instincts dans toutes les circonstances de la vie et donnent un aspect de pleine réalité à des espoirs d'actions prochaines. (Ibid.)

\section{L'ÉTONNANT POUVOIR DE CES RÊVES MATÉRIALISÉS QUE L'ON APPELLE HYPOICÔNES}

Le signe que Peirce appelle hypoicône transforme l'aspect le plus évanescent de la sémiose en sinsignes concrétisés, compréhensibles, en choses dont la seule présence suffit à montrer qu'un état du monde possible, imaginaire, se réalise ici et maintenant. L'existence tangible de l'hypoicône ne peut être niée ou laissée de côté (comme c'est le cas pour un effet de l'imagination). Les éléments proposés par Anderson comme fondement imaginaire d'une véritable communauté nationale - ces icônes à double référent initialement prévues comme signes de la soumission des colonisés à la règle du colon européen - et le mythe décrit par Sorel dans sa discussion de la lutte sociale qui vise à renverser l'ordre établi, tous portent la marque de la double puissance sémiotique de la qualité pure et de l'existence tangible à laquelle s'attache la généralité symbolique. C'est l'attrait de l'hypoicône éternelle qui inquiète maintenant les humanistes, qui voient dans la télévision et les images des jeux vidéo l'absence éloquente et menaçante de la parole et des arguments qu'elle construit.

Dans ce bref voyage à travers les communautés imaginées et les rêves de grèves générales, nous espérons avoir montré comment le fonctionnement transparent des hypoicônes est un trait immanent à la vie de l'humanité, aussi vital et incontournable pour la sémiose que ses deux autres composantes, puisque toutes à leur façon peuvent mener (et mènent effectivement) à de violents soulèvements sociaux, à des guerres d'indépendance ou aux superproductions mondialisées de MTV. En l'absence des hypoicônes, les objets tangibles du monde et ceux de notre imagination resteraient à jamais dissimulés par le voile intangible du Ding an sich, notion même que la sémiotique de Peirce s'efforce de combattre. La tension fluctuante qui va de la dimension sémiotique virtuelle et onirique à la manifestation concrète, 
historique, de la relation de ressemblance établie au moyen des hypoicônes est ce qui explique la puissance de l'imagination humaine et de sa capacité infinie à présenter de nouvelles qualités inconnues, qui seront matérialisées et mises en œuvre au moyen de lois générales, symboliques.

La présente recherche a été rendue possible grâce au soutien de la Fondation Alexander von Humboldt Stiftung à Bonn.

\section{NOTES}

1. Liszka, 1996: 46. Liszka propose également une «règle d'inclusion", selon laquelle «l'indice permet au symbole de référer alors que l'icône lui permet de signifier" (ibid.).

2. Les citations renvoient au texte remanié par Ransdell en 1997, et disponible sur le site du Peirce Telecommunity Project sous forme de 53 "paragraphes".

Afin de faciliter la lecture, le lecteur prendra note que tous les passages cités ont été traduits en français. On peut facilement recourir à la version anglaise puisque la pagination donnée entre parenthèses renvoie au texte original (N.D.T.).

3. Jusqu'à nouvel ordre, les citations de Peirce proviennent des paragraphes 1.341-342 des Collected Papers.

4. Cette expression "elle est ce qu'elle veut", à caractère biblique, revient dans un texte tardif très important de Peirce qui traite du musement (C.P. 6.548)

5. Peirce décrit la signification des verbes comme « un simple rêve, un produit de l'imagination sans attaches avec une situation particulière susceptible d'évoquer une icône». Détaché analytiquement d'un indice, le verbe ne possède rien d'autre que son pouvoir de produire des rêves (cf. C.P. 4.56).

6. L'exemple du tableau est utilisé par Peirce lorsqu'il introduit la notion d'hypoicône.

7. «Ce qui manque [à une grenouille décapitée], c'est le pouvoir de la méditation préparatrice» (C.P. 6.286).
8. «C'est ainsi que le journal de Caracas a tout naturellement et apolitiquement créé une communauté imaginée parmi un regroupement de lecteurs communs». Anderson (1993: 62).

9. C'est également la position de Gellner, cité par Anderson.

10. Ce qui semble le plus important à Anderson pour la genèse des "communautés imaginées" (1993: 133), c'est la langue sous sa forme imprimée plutôt que l'idée de la langue fédératrice comme le soutiennent les idéologies nationalistes.

11. Le terme plutôt bon enfant d'«unissonance» a été créé par Anderson (1993: 145)

12. Il est intéressant de noter que l'on peut dire à peu près la même chose du syncrétisme religieux que nous avons rencontré à Bahia au nord du Brésil. La religion catholique, qui avait été imposée aux noirs arrivés comme esclaves en Amérique, fut adoptée mais iconiquement transformée par les nouveaux arrivants. Sur les hypoicônes de l'iconographie judéo-chrétienne, la Vierge Marie, par exemple, ils greffèrent l'icône de leurs propres dieux, par exemple la déesse de la mer, Jemanjá. Loin de rejeter la foi reçue, ils la transformèrent iconiquement jusqu'à l'obtention de nouvelles hypoicônes. De nos jours, dans les temples Umbanda, on peut apprécier la riche association des deux hypoicônes, la catholique et l'afro-américaine, comme dans un palimpseste complexe.

\section{RÉFÉRENCES BIBLIOGRAPHIQUES}

AlEXANDER, T. [1990]: «Pragmatic Imagination », Transactions of the C.S. Peirce Society, vol. XXVI, 3, 325-348.

ANDACHT, F. [1996]: « El lugar de la imaginación en la semiótica de C.S. Peirce ", Anuario Filosófico, vol.XXIX, 3, 1265-1290.

ANDERSON, B. [1983]: Imagined Communities. Reflections on the Origin and Spread of Nationalism, London, Verso, 1993.

LIsZKA, J.J. [1996]: The Semiotic of Charles S. Peirce, Bloomington, Indiana University Press.

PeIRCE, C. S. [1931-35]: Collected Papers, vol. I-VI (sous la dir. de C. Hartshorne et P. Weiss), Cambridge, Mass., The Belknap Press;

[1958]: Collected Papers, vol. VII-VIII (sous la dir. de A. W. Burks), Cambridge, Mass., The Belknap Press.

RANSDELL, J. [1979]: "The Epistemic Function of Iconicity in

Perception ", Peirce Studies 1, Institute for Studies in Pragmaticism, 51-66;

[1986]: «On Peirce's Concept of Iconic Sign», Iconicity. Essays on the Nature of Culture (sous la dir. de P. Bouissac et al.), Tübingen, Stauffenverlag. (Cité d'après la version révisée de 1997, disponible au Peirce Telecommunity Project [http://www.door.net/arisbe].)

SOREL, G. [1908]: Réflexions sur la violence, Paris, Imprimerie nationale. 